\title{
Formación Dual en Grados de Maestro: Feedback Interactivo en la Evaluación por Competencias
}

\section{Dual Training in Teacher's Degrees: Interactive Feedback in the Competences Assessment}

\author{
M. Inés Gabari Gambarte * \\ Jaione Apalategi Begiristain
}

Universidad Pública de Navarra/Nafarroako Unibertsitate Publikoa, España

\begin{abstract}
La evaluación por competencias en el espacio de educación superior que introdujo el llamado proceso de Bolonia sigue siendo un reto al se enfrenta la institución universitaria desde hace dos décadas. La propuesta de buenas prácticas en la Formación inicial de los Grados de Maestro, recogida en este artículo, se plantea el objetivo de implementar un proceso formativo dual universidad-colegios de Educación Primaria como escenario para el desarrollo y evaluación de las competencias específicas de la asignatura Procesos y Contextos Educativos/Hezkuntza Prozesuak eta Testuinguruak ofertada en segundo curso del Plan de Estudios en diversas lenguas (castellano y euskera -lenguas oficiales- e inglés) en la Universidad Pública de Navarra/Nafarroako Unibertsitate Publikoa. Se opta por un diseño de alternancia exterior asociativa que se ha ido consolidando en los últimos seis cursos y en el que han participado 400 estudiantes universitarios. Los resultados describen la secuencia lógica de las etapas, medios, técnicas e instrumentos de evaluación, así como la participación de los diferentes agentes implicados en la autorregulación de los aprendizajes competenciales, para que se trate de una evaluación auténtica, participativa y compartida. Las conclusiones apuntan a la importancia de crear espacios de alternancia que conecten los aprendizajes universitarios con la práctica contextualizada, para que respondan a las demandas sociales y que, a su vez, conlleven la debida acreditación de los niveles de competencia adquiridos.
\end{abstract}

Palabras clave: Formación dual; Buenas prácticas; Evaluación competencias; Educación superior; Evaluación auténtica; Feedback.

The evaluation by competences in the space of higher education that introduced the socalled Bologna process continues to be a challenge faced by the university institution for two decades. The proposal of good practices in the Initial Training of the Degrees of Master, collected in this article, aims to implement a dual university-primary school training process as a scenario for the development and evaluation of the specific competences of the subject Processes and Educational Contexts/Hezkuntza Prozesuak eta Testuinguruak offered in the second school year of the curriculum in various languages (Spanish and Basque -official languages- and English) at the Public University of Navarra/Nafarroako Unibertsitate Publikoa. We opt for an associative exterior design that has been consolidated in the last six years and in which 400 university students have participated. The results describe the logical sequence of the stages, means, techniques and evaluation instruments, as well as the participation of the different agents involved in the self-regulation of competency learning, so that it is an authentic, participatory and shared evaluation. The conclusions point to the importance of creating spaces of alternation that connect university learning with contextualized practice, so that they respond to social demands and that, in turn, entail the proper accreditation of the levels of competence acquired.

Keywords: Dual training; Good practice; Evaluation of competences; Higher education; Authentic evaluation; Feedback.

*Contacto: igabari@unavarra.es

issn: 1989-0397

www.rinace.net/riee/

https://revistas.uam.es/riee
Recibido: $\quad 10$ de mayo de 2019

$1^{\text {a }}$ Evaluación: 29 de julio de 2019

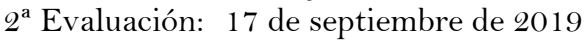

Aceptado: $\quad 22$ de septiembre de 2019 


\section{Introducción}

La sociedad actual es cada vez más móvil y digital y su complejidad es tremendamente mutante. Al respecto, es oportuno referir la aportación que desde la sociología nos ha dejado como valioso legado Zygmunt Bauman, con su célebre concepto de "sociedad líquida” acuñada en 1998 (Bauman, 2015). Con ella definió a la sociedad contemporánea como un mundo que se encuentra en constante movimiento, inestable y atomizado, dónde los individuos están sometidos a una competición frenética y, sin embargo, son los únicos responsables de su existencia. Para este pensador neoliberal, la era consumista de "todo es desechable" prepara para aceptar lo inaceptable y para desolidarizarse de toda clase de vidas ruinosas.

Recientemente, Lemonnier (2019) se ha referido a un manuscrito que el autor, desaparecido en 2017, realizó bajo el título de Retrostopia, nombre con el que se ha editado un libro póstumamente. En esta obra Bauman invita a los individuos a superar todos los miedos que operan en la sociedad contemporánea para así poder crear, urgentemente, nuevas utopías.

Públicamente se observa un acuerdo mayoritario en torno a la idea de que, para poder vivir activamente en la sociedad actual, se requieren personas cualificadas y competentes creativamente, críticas, resueltas y determinativas, al tiempo que se cuestiona cómo afrontar y conseguir este reto si no somos capaces de crear y adaptar modos flexibles de aprendizaje, formación y educación (Consejo Comisión de Europa, 2018).

En relación con lo anterior, el documento de reflexión sobre la dimensión social de Europa (Comisión-Europea, 2017) señala que existe unanimidad sobre la idea de que las circunstancias que más influirán en el futuro de nuestras sociedades y economías son la educación y las competencias. De modo generalizado se admite que en Europa existen algunos de los sistemas educativos más avanzados e innovadores del mundo. Sin embargo, resulta desalentador el dato de que alrededor de un cuarto de su población adulta, por ejemplo, tenga un nivel insuficiente de lectura y escritura, y que casi la mitad carezca de competencias digitales suficientes. Lo que es aún peor, es que se constata un desempeño cada vez más deficitario entre los jóvenes en materia de competencias básicas.

Igualmente, se constata que la marcha de la digitalización afecta holísticamente en nuestras vidas y, por ende, en la evolución económica, dónde se desplazarán algunos de los empleos actuales por otros aún desconocidos que requerirán de nuevas competencias para su ejercicio. No es fácil predecir todos los efectos de la automatización y de la inteligencia artificial en el nicho laboral futuro, pero es evidente que, para intentar superarlo, entre otros, es imprescindible adaptar los sistemas educativos.

\section{Revisión de la literatura}

Estudios recientes señalan que de aquí a mediados del siglo XXI, la mitad de las actividades profesionales actuales podrían estar automatizadas y ello planteará nuevos retos a la educación, formación, competencias y aprendizaje en el ciclo vital, gestión de los recursos humanos y capacidad para gestionar transiciones profesionales complejas a lo largo de la vida de una persona (Espinoza Freire, Tinoco Izquierdo y Sánchez Barreto, 2017). "Lo cierto es que los trabajadores de todas las edades se verán obligados cada vez con mayor frecuencia a adaptar sus competencias al cambio tecnológico y a actualizarlas 
continuamente" (Comisión-Europea, 2017, p. 18), y ello supone, incuestionablemente, la implicación directa de las instituciones educativas en general, y las de educación superior en particular, las cuales deberán preparar a sus estudiantes hacia este escenario de futuro (ver Figura 1).

\section{Retos para la vida laboral de mañana}

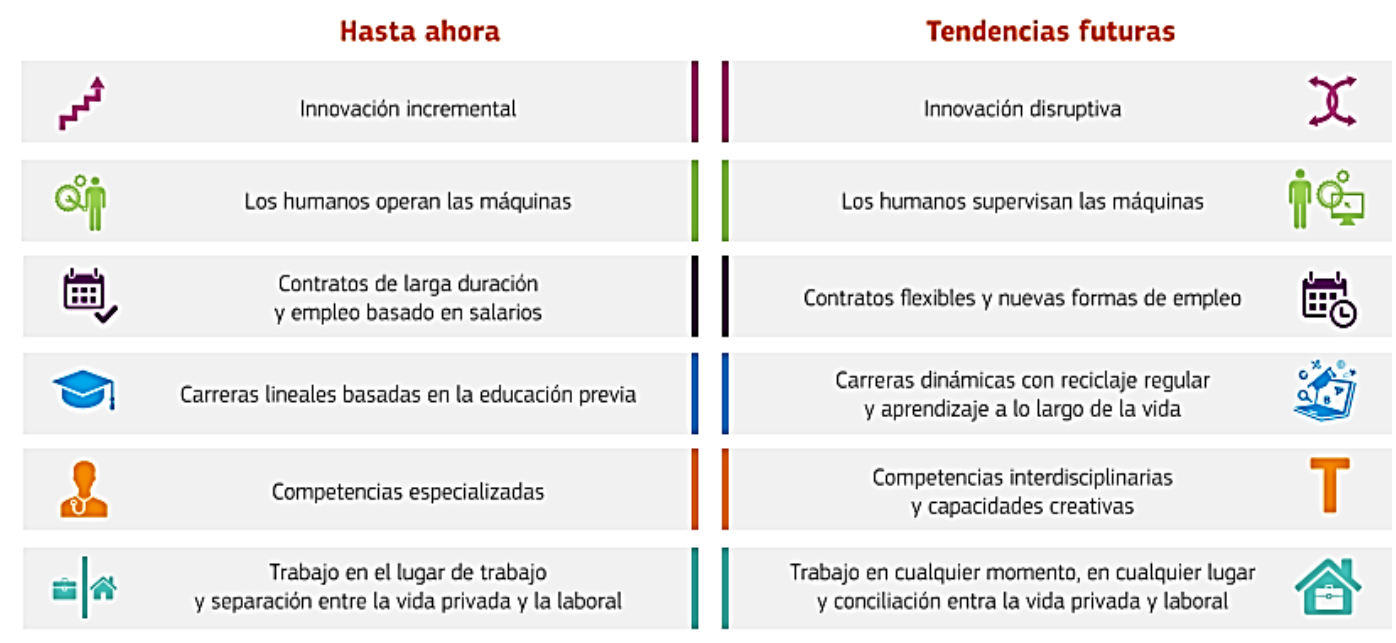

Figura 1. Retos para la vida laboral de mañana Fuente: Comisión Europea (2017, p. 18).

En lo que implica al ámbito de la educación superior, este reto supone desarrollar una formación inicial de calidad y la evaluación por competencias es la apuesta ganadora de esta partida. Y es que la evaluación, juega un rol fundamental en la estructuración de los procesos de aprendizaje y de formación, validando "buenas prácticas" en torno a nuevas formas de enseñanza (Consejo Comisión Europea, 2018). Son cada vez más, las instancias internacionales de educación superior que diseñan y desarrollan tanto sus Grados como sus Postgrados, apoyados en dispositivos duales o en alternancia (Beraza y Azkue, 2018)

Dentro de la temática de Evaluación por competencias en el ámbito de la Educación Superior, coincidimos con Cano (2015, p. 81) cuando afirma que:

La evaluación de los aprendizajes ha de entenderse como un proceso en el que se recoge información acerca de la evolución y de las ejecuciones de los estudiantes y se valora a la luz de unos criterios conocidos y relevantes para poder tomar decisiones.

De igual manera, estamos de acuerdo con esta autora, cuando a partir de la revisión de la literatura que realiza sobre las características del proceso de evaluación, afirma que una buena práctica evaluativa es buena, sólo, cuando es congruente con los objetivos, contenidos y metodología, cuando es diversa, cuando implica a diferentes agentes y cuando permite la autorregulación de los aprendizajes (Cano, 2011).

Pascual-Arias, López-Pastor y Hamodi-Galán (2019) recogen las propuestas de numerosos autores que coinciden en afirmar que la evaluación formativa es el proceso de evaluación que tiene como finalidad la mejora del proceso de enseñanza-aprendizaje. Para que se produzca esta mejora, la evaluación formativa debe abarcar tres dimensiones: a) el aprendizaje del alumnado, b) el proceso de enseñanza del docente y c) el proceso de enseñanza-aprendizaje. Se trata de la denominada evaluación continua dado que se va 
produciendo a lo largo del proceso y permite rectificaciones y optimizaciones de manera simultánea al transcurso de la acción-reflexión.

Si al momento y a la finalidad de la evaluación le añadimos el agente que la lleva a cabo, es de obligada referencia incluir la evaluación compartida, entendida como el proceso de valoración que incluye al propio estudiante. En este punto es oportuno enlazar ambos conceptos de evaluación 'formativa' y 'compartida' con las nociones de Cano (2015, p. 169) referidas al feedback y al feedfodward en el sentido de que para que la evaluación sea formativa, optimizante, es decir, para que se dé un verdadero feedfodward es imprescindible que también sea compartida, tal y como afirma la autora.

Se aboga, pues, como indican Allal y López (2005), por un feedback co-construido, espiral, que cierre el círculo del aprendizaje con su aplicación en futuras tareas y que sea adaptable y contextualizado. Este feedback interactivo que ha de aplicarse a tareas futuras es el feedfodward.

Una de las recientes publicaciones de Le Boterf (2018) en torno a cómo desarrollar e implementar competencias se dirige a los gestores de recursos humanos empresariales u organizativos. Sus aportaciones pueden hacerse extensibles al ámbito de la gestión de la enseñanza superior, ya que una de sus misiones es preparar a los estudiantes para que actúen como profesionales competentes. Así, nos parece pertinente la aclaración de partida que realiza en el primer capítulo del libro, en torno a los conceptos de "profesionalismo", entendido como el modo de ganarse la vida en cualquiera de sus cuatro niveles: debutante, profesional confirmado y experto, en contraposición al de "profesionalización" entendido como la acción de formar o formarse.

Además, frente al uso indiscriminado y escasamente unívoco del concepto de competencia, resulta valiosa su propuesta de definición dual: "être compétente" o "ser competente" como proceso de saber cómo actuar en una situación profesional mediante la movilización de una combinación adecuada de recursos internos (conocimiento, habilidades, aptitudes, emociones, ...) y recursos externos (recursos del medio ambiente) y, "avoir de compétences" o "tener competencias", como recurso personal o combinación de recursos personales necesarios para actuar en una situación laboral. De cualquier modo, el eje en torno al cual se genera el desarrollo competencial es la capacidad reflexiva. Esta reflexividad es la que pone al profesional en el corazón de su propio desarrollo profesional y de la implementación de su profesionalismo como responsabilidad compartida que es.

La definición dual de competencia de Le Boterf pone el acento en que la sociedad que acoge a los titulados de las instituciones de la enseñanza superior debe formarlos poniendo a los estudiantes en el centro de su aprendizaje y de su evaluación competencial, evidentemente, en asociación con el profesorado. Todo ello directamente relacionado con las nociones de evaluación formativa y compartida así como con la de feedfodward, mencionadas en líneas precedentes.

Según Lemenu y Heinen (2015) en relación con la calidad de la formación inicial del profesorado, la evaluación de las prácticas de enseñanza, en particular las implementadas durante las prácticas en programas de trabajo y estudio, revela importantes desafíos. Esta evaluación, para ser formativa, requiere una evidencia y un rigor tan sólidos como la evaluación de certificación que valida el acceso a la profesión docente. Indudablemente este proceso evaluador pasa por el logro de un consenso intersubjetivo entre los agentes 
implicados, lo que vuelve a enlazar con la visión de feedfodward en la evaluación por competencias.

A modo de cierre de este apartado y en coincidencia con las ideas expuestas, consideramos oportuno referir la aportación de Loisy (2016) de la Universidad de Lyon, investigadora en desarrollo profesional y construcción de la función docente, realizada a través del Canat-U de C.R.A.I.E.S ${ }^{1}$ dónde defiende que: la noción de evaluación de competencias sólo puede efectuarse en una situación de evaluación que permita movilizar efectivamente las competencias, preferentemente desde un enfoque dinámico e interactivo. Además, existe una necesidad de alineación entre competencias denominadas objetivos de aprendizaje, las situaciones implementadas para desarrollar estas competencias y las situaciones de evaluación.

\section{Método}

El objetivo general es implementar un proceso formativo dual universidad-colegios de Educación Primaria (EP) que sirva como escenario para el desarrollo y evaluación de las competencias específicas de la asignatura Procesos y Contextos Educativos/Hezkuntza Prozesuak eta Testuinguruak ofertada en diversas lenguas vehiculares en el segundo curso (semestre otoño) del Plan de Estudios del Grado de Maestro EP en la Universidad Pública de Navarra/Nafarroako Unibertsitate Publikoa (cuadro 1).

Los objetivos específicos son: 1) diseñar medios, técnicas e instrumentos de recogida de evaluación continua y formativa, 2) articular procesos de feedback y feedforward (Cano, 2015) de reflexión, participación y mejora en el proceso de enseñanza-aprendizaje y 3) optimizar la relación teórico-práctica de los saberes profesionales docentes en alternancia aula universitaria-contexto real de aula escolar.

\subsection{Diseño}

La formación dual o en alternancia es entendida por el Centro Europeo para el Desarrollo de la Formación Profesional (Cedecop) como la educación o aprendizaje que alterna períodos en una institución educativa o en un taller de aprendizaje con el lugar de trabajo (Beraza y Azkue, 2018, p. 55). En concreto se plantea un diseño en alternancia asociativa externa Universidad-Colegio en la etapa de Educación Primaria. Esta dualidad se diferencia de otros tipos en que, según Bourgeon (1979) supone la "Asociación entre formación profesional y formación general en la que se da una adición entre estudios y actividades" (citado por Tejada, 2012, p.30), lo que recogemos de manera más detallada en otra publicación sobre el tema (Apalategi y Gabari, 2018, p. 328) y que se sintetiza a continuación (ver cuadro 2).

\footnotetext{
${ }^{1}$ Conception de Ressources pour l'Approche-programme: Innovation dans l'Enseignements Superieur) el 8 de marzo de 2016 , https://www.canalu.tv/video/ecole_normale_superieure_de_lyon/approche_programme_approc he_par_competences_et_d_autres_notions_associees.20844
} 
Cuadro 1. Competencias Específicas: Procesos y Contextos Educativos/Hezkuntza Prozesuak eta Testuinguruak

Conocer los objetivos, los contenidos Adquirir hábitos y destrezas de curriculares, el significado de las áreas y

1. la organización, la metodología y los criterios de evaluación de la Educación Primaria

9. para favorecer la implicación activa del alumnado en su desarrollo social y personal.

Diseñar, planificar y evaluar los procesos

9. de enseñanza y de aprendizaje atendiendo a criterios interdisciplinares y disciplinares con otros profesionales

Reflexionar en relación a las prácticas de aula para innovar y mejorar la labor

10. docente, y referirlas al funcionamiento de los procesos psicológicos básicos, a los modelos pedagógicos y a los criterios disciplinares de la etapa.

Diseñar y regular espacios de aprendizaje en contextos de diversidad,

multiculturales y plurilingües. Atender

las necesidades singulares del alumnado, la igualdad de género, la equidad, el respeto y los derechos humanos.

Conocer las implicaciones educativas de

11. las tecnologías de la información y la comunicación.

Profundizar en los contextos de aprendizaje y de convivencia escolar, la

5. aceptación de normas, la constancia, la disciplina personal y el respeto a los demás.

Conocer la organización de los centros de educación primaria y su funcionamiento

6. en colaboración con los distintos sectores de la comunidad educativa y del entorno social.

Organizar de forma activa los procesos de enseñanza y de aprendizaje de los contenidos de la Educación Primaria

12. desde una perspectiva de desarrollo de competencias. Conocer modelos de mejora de la calidad

Desempeñar las funciones de tutoría, de 13. orientación y de resolución de conflictos con el alumnado y sus familias. Saber observar y validar el trabajo bien hecho.

Contextualizar la acción docente ante los

Fomentar la cooperación, la motivación y

7. el deseo de aprender, y participar cambios políticos, sociales, y pedagógicos, fomentar la educación activamente en los proyectos de centro.

democrática y el desarrollo de una ciudadanía activa para la consecución de un futuro sostenible.

Mantener una relación crítica y autónoma

8. respecto de los saberes, los valores y las instituciones implicadas en la educación.

Fuente: Elaboración propia.

El alumnado universitario acude con una periodicidad de una/dos sesiones semanales de dos horas/sesión a un aula de un colegio teniendo la oportunidad de:

1. Desempeñar el rol de docencia compartida

2. Articular el aprendizaje y diseño de actividades didácticas con metodologías activas (en el aula universitaria) y el desarrollo de las propuestas en el contexto real de enseñanza-aprendizaje

3. Reflexionar sobre todo el proceso, recibir retroalimentación y mejorar sus aprendizajes en la praxis. 
Cuadro 2. Tipologías de alternancia

\begin{tabular}{|c|c|c|c|c|c|}
\hline \multicolumn{2}{|r|}{ GIROD (1974) } & \multicolumn{2}{|c|}{ MALGLAIVE (1979) } & \multicolumn{2}{|c|}{ BOURGEON (1979) } \\
\hline $\begin{array}{l}\text { Iี } \\
\text { Iี }\end{array}$ & $\begin{array}{l}\text { Acercamiento del } \\
\text { mundo intelectual } \\
\text { (educativo) a } \\
\text { adultos o jóvenes } \\
\text { que tienen } \\
\text { experiencia de } \\
\text { trabajo }\end{array}$ & $\frac{\vec{d}}{\tilde{z}}$ & $\begin{array}{l}\text { Durante la formación } \\
\text { Espacios libres sin } \\
\text { relación entre } \\
\text { formación académico } \\
\text { y actividades prácticas }\end{array}$ & 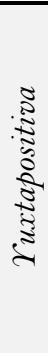 & $\begin{array}{l}\text { Periodos } \\
\text { intercalados de } \\
\text { actividades de } \\
\text { estudio y trabajo } \\
\text { sin relación } \\
\text { aparente. } \\
\text { Disyunción } \\
\text { institucional y } \\
\text { didáctica }\end{array}$ \\
\hline \multirow[t]{2}{*}{ 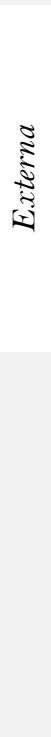 } & $\begin{array}{l}\text { Fijación de } \\
\text { periodos de trabajo } \\
\text { durante los estudios }\end{array}$ & 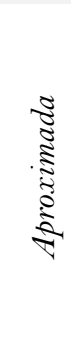 & $\begin{array}{l}\text { Organización } \\
\text { didáctica que une de } \\
\text { manera coherente } \\
\text { tiempos y espacios de } \\
\text { formación. } \\
\text { Observación y análisis } \\
\text { de la realidad, sin } \\
\text { intervención }\end{array}$ & 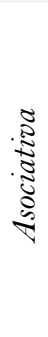 & $\begin{array}{l}\text { Asociación entre } \\
\text { formación } \\
\text { profesional y } \\
\text { formación general. } \\
\text { Adición de estudios } \\
\text { y actividades } \\
\text { profesionales sin } \\
\text { transversalidad. }\end{array}$ \\
\hline & & $\begin{array}{l}\widetilde{\Xi} \\
\approx\end{array}$ & $\begin{array}{l}\text { Formación teórica y } \\
\text { práctica global. El } \\
\text { alumnado construye y } \\
\text { pone en práctica su } \\
\text { propio proyecto } \\
\text { pedagógico y lo } \\
\text { analiza } \\
\text { reflexivamente. } \\
\text { Gran implicación de } \\
\text { las instituciones }\end{array}$ & $\frac{\sqrt{8}}{\frac{3}{3}}$ & $\begin{array}{l}\text { Compenetración } \\
\text { efectiva entre los } \\
\text { medios de vida } \\
\text { socioprofesional y } \\
\text { escolar en una } \\
\text { unidad de tiempo } \\
\text { formativa. }\end{array}$ \\
\hline
\end{tabular}

Fuente: Adaptado de Tejada (2012).

\subsection{Participantes}

En el curso 2013-2014 el grupo de mañana del Grado de Maestro EP, que cursa los estudios en euskera como lengua vehicular, lleva a cabo una primera experiencia de formación dual asociativa para poder implementar los diseños didácticos elaborados en el aula universitaria en contextos reales de EP y, consecuentemente, poder evaluar el aprendizaje competencial del alumnado universitario en relación con la asignatura (Competencias específicas).

A partir de esta experiencia, se va consolidando la propuesta y se hace extensiva a otros grupos de alumnos y alumnas, de los turnos de tarde y a aquellos que cursan los estudios en otras lenguas vehiculares como castellano e inglés.

Las formas de participación van formulándose en un abanico variado, en función de criterios como:

1) Momento inicial: la selección previa, por parte de la docente de la universidad, en función de la calidad de las propuestas didácticas con metodologías activas diseñadas en el aula universitaria

2) Posteriormente: las necesidades expresadas por los centros que demandan el diseño de propuestas didácticas para sus aulas incluyendo determinadas metodologías en relación con el aprendizaje cooperativo 
La relación de los colegios que solicitan participar se va incrementando cada curso, de modo que hasta el momento han participado:

- por parte de la universidad:

○ un número total de 400 estudiantes

$\bigcirc$ un equipo de ocho docentes universitarios

- por parte de los colegios Educación Infantil-Educación Primaria:

- 11 centros con modelos lingüísticos: D (enseñanza en euskera), G (enseñanza en castellano), A (enseñanza en castellano con asignatura euskera), PAI (Programa de Aprendizaje en Inglés) y British (enseñanza en inglés) https://www.educacion.navarra.es/web/dpto/idiomasplurilinguismo

○ 50 maestros y maestras

El alumnado universitario se incorpora a las actividades escolares durante horario lectivo, lo que supone un esfuerzo organizativo por parte del profesorado universitario que tiene que velar por articular los periodos de clase de la institución de educación superior con los de la diversidad de los colegios. Se formaliza por escrito a priori un compromiso que garantiza la posibilidad de asistencia presencial a las sesiones de todas de las asignaturas del Grado, de modo que no haya interferencia entre franjas horarias de clase en la UPNA/NUP y franja horaria de las dos sesiones semanales de participación en el aula escolar.

\subsection{Instrumentos}

Para que la formación dual sea el contexto previsto de desarrollo de competencias profesionales y su evaluación, es preciso emplear numerosos instrumentos elaborados ad hoc, tanto a nivel de auto y coevaluación del alumnado como de heteroevaluación por parte los dos colectivos de docentes (universitarios y escolares) de los logros evidenciados por los estudiantes en formación, para intercambiar datos que se puedan analizar e interpretar de cara a la mejora (evaluación formativa), entre otros están:

- Los empleados con los/las profesionales de los centros escolares:

$\checkmark$ Entrevistas presenciales con Equipos Directivos y responsables de innovación

$\checkmark$ Correos electrónicos con los maestros y maestras en cuyas aulas interviene el alumnado universitario

$\checkmark$ Plataformas Drive interactivas para calendarizar sesiones y franjas horarias

$\checkmark$ Anecdotarios para recoger incidencias fortuitas

$\checkmark \quad$ Informes para reflejar el nivel de la progresión de los aprendizajes

$\checkmark$ Certificados para acreditar la validez del aprendizaje

- Los empleados con el alumnado, tanto a nivel individual como de pequeño-grupo (p-g) de trabajo cooperativo:

$\checkmark$ Protocolos diversos: a) Compromiso de Confidencialidad, c) Certificado negativo de delitos sexuales, d) Abono de seguro escolar 
$\checkmark$ Escalas de estimación

$\checkmark$ Fichas-registro de datos cuantitativos y cualitativos (narrativos)

$\checkmark$ Rúbricas valorativas

$\checkmark$ Producciones: parciales (tareas, actividades, etc.) y finales (trabajos, exposiciones, portafolios, etc.)

$\checkmark$ Videograbaciones

$\checkmark$ Entrevistas de tutorización y seguimiento continuo de los estudiantes universitarios antes, durante y tras su participación en el contexto educativo dual.

\subsection{Procedimiento}

Al inicio de curso, mes de septiembre, se lleva a cabo una reunión inicial entre profesorado universitario y equipos directivos y docentes de cada colegio que ha formulado la demanda y/o disponibilidad. Se crea una plataforma Google Drive compartida entre las dos instituciones para facilitar la elección de franjas horarias días de la semana y lengua en la que se desarrollan las sesiones escolares (castellano, euskera, inglés). Cada alumno/a se responsabiliza de la entrega de los protocolos requeridos para poder acceder al centro escolar. El alumnado se incorpora a las sesiones desde mitades de septiembre o inicios de octubre hasta finalizar el semestre. De forma paralela comienza el desarrollo del tiempo lectivo y las clases en la universidad, en las que se trabajará individualmente, en pequeñogrupo y en grupo-aula en los diseños didácticos y demás contenidos de la asignatura.

A lo largo del proceso se mantiene una comunicación fluida entre profesorado universitario y maestros/maestras escolares, en su mayoría de manera virtual (por correos electrónicos o red social). Durante el mes de diciembre se celebran de nuevo entrevistas presenciales de evaluación compartida y se solicita al alumnado la autoevaluación individual de su aprendizaje competencial, procediendo a la triangulación de la información. En el mes de enero se formalizan los informes y las acreditaciones y se hacen llegar al alumnado. En el mes de febrero se lleva a cabo la elaboración del informe conjunto de asignatura en todos los grupos en los que se imparte y se entrega por la responsable a la Facultad de Ciencias Humanas y Sociales.

\section{Resultados}

La implementación de la experiencia ha requerido una flexibilidad y un proceso cíclicoreflexivo similar al seguido por las investigaciones transformativas de la investigaciónacción (Colmenares y Piñero, 2008). La carencia de una formalización previa de estructura dual ha supuesto que los procesos de aprendizaje y las herramientas para evaluarlos hayan ido emergiendo conforme se iba dando la complejidad que requiere la articulación entre los aprendizajes y su contextualización en la realidad (Fernández y Bueno, 2016).

Para abordar el aprendizaje competencial, dejando a un lado el debate de si es o no posible enseñar competencias o si tan solo se pueden desarrollar (Zabala y Arnau, 2014), se han diseñado diversas situaciones de aprendizaje que respondieran a los criterios de enseñanza de las que el Plan de Estudios atribuye a la asignatura, haciendo más hincapié en las específicas (CE). En primer lugar el Criterio de Significatividad nos condujo a la necesidad de partir de los conocimientos previos del estudiante universitario y a la exigencia de 
presentar los contenidos de forma funcional, tomando como referencia su propio nivel de desarrollo para provocarles un conflicto cognitivo que tuvieran que resolver con la actividad mental precisa, a la vez motivase una actitud favorable estimuladora de su autoestima, reforzadora de su autoconcepto y que sirviese como herramienta para a aprender a aprender por sí solos/solas (Zabala y Arnau, 2008).

Decidimos introducir una actividad de sensibilización terminológico-conceptual mediante una Lectura Dialógica (en conjunto refleja un 25\% de la calificación final sumativa) por cada uno de los ocho temas del Programa que supusiera: 1) una lectura individual autónoma fuera del aula universitaria, con elección de una cita justificada en función de los intereses, grados de acuerdo/desacuerdo, etc. y ya en el aula universitaria 2) un trabajo dialógico grupal en pequeños-grupos (p-g) cooperativos de cuatro componentes (20'), 3) un proceso de selección de la aportación más representativa del grupo, 4) la defensa oral de la toma de decisiones por parte de un componente que asume el rol de responsable (rotario cada semana no pudiendo desempeñarse en semanas contiguas) y 5) una auto y coevaluación del desarrollo del proceso (feedback) que cumplimenta y entrega al final de la sesión el/la responsable y que irá reflejándose en el porcentaje. La devolución de feedforward (Ion, Silva y Cano, 2013) por parte de la docente se realiza tanto durante la defensa oral de la cita grupal como por escrito en el propio registro que se devuelve comentado (ver figura 2).

\begin{tabular}{|c|c|c|c|}
\hline \multicolumn{4}{|c|}{ LECTURAS DIALOGICAS (SOBRE 2,5 PUNTOS/8 SESIONES) } \\
\hline \multicolumn{4}{|l|}{ TÍTULO OBRA_ } \\
\hline $\begin{array}{c}\text { NOMBRE Y } \\
\text { FIRMA } \\
\text { COMPONENTES } \\
\text { GRUPO } \\
\end{array}$ & $\begin{array}{c}\text { COEVALUACION } \\
\text { 0-CONTRIBUCION NULA } \\
\text { 1-CONTRIBUCION MEDIA } \\
\text { 2-CONTRIBUCION EXCELENTE }\end{array}$ & $\begin{array}{c}\text { CITA SELECCIONADA } \\
\text { POR } \\
\text { COMPONENTE/PAGINA }\end{array}$ & $\begin{array}{l}\text { CITA } \\
\text { SELECCIINADA } \\
\text { POR EL P-G }\end{array}$ \\
\hline \multicolumn{4}{|c|}{ 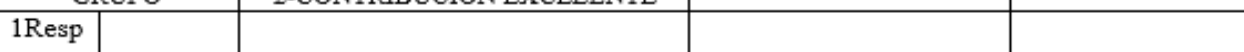 } \\
\hline \multicolumn{4}{|l|}{2} \\
\hline \multicolumn{4}{|l|}{3} \\
\hline \multicolumn{4}{|l|}{4} \\
\hline \multicolumn{4}{|c|}{ REFLEXIÓN Y DEFENSA FINAL $\left({ }^{*}\right)$ : } \\
\hline \multicolumn{4}{|c|}{$\begin{array}{l}\text { (*) CADA COMPONENTE ACTUARÁ COMO RESPONSABLE DE LA ENTREGA DE DOS } \\
\text { LECTURAS DIALÓGICAS NO CONTIGUAS EN FECHA }\end{array}$} \\
\hline \multicolumn{4}{|c|}{ Nombre legible y firma del responsable de la lectura dialógica: $L$} \\
\hline
\end{tabular}

Figura 2. Ficha registro Lectura Dialógica

Fuente: Elaboración propia.

La reflexión sobre el Criterio del Carácter procedimental de la acción competente, nos llevó a formular un trabajo de p-g cooperativo sobre el estudio de metodologías activas que, posteriormente, tendrían que incorporar al diseño de propuestas didácticas en contexto real. La acción competente implica un saber hacer que precisa destrezas y habilidades encadenadas desde el punto de partida con actividades funcionales, pasando por modelos de acciones en las que se vislumbren todas las fases, en un proceso gradual, con diversidad de recursos de ayuda y con una praxis individualizada, por lo que en esta actividad los pasos a seguir serán: 1) consulta de diversas fuentes facilitadas por la docente 
sobre la metodología elegida por el p-g, 2) labor de síntesis (siguiendo un guión), 3) exposición oral en p-g (30’) y elaboración de un material de estudio para el grupo-aula y 4) diseño-desarrollo en el aula universitaria de una tarea de clase que incorpore la metodología.

La evaluación en este caso vuelve a darse a dos niveles. A través de una escala de estimación cada p-g valora los siete aspectos de la exposición requeridos en el Guión (ficha técnica de referencia bibliográfica, concepto/autores, características, aplicaciones a la enseñanza, ventajas e inconvenientes de su empleo), incluida la aplicación a una situación en el contexto real del aula universitaria (ejemplos), lo que supone una coevaluación entre iguales a nivel de feedback (ver figura 3).

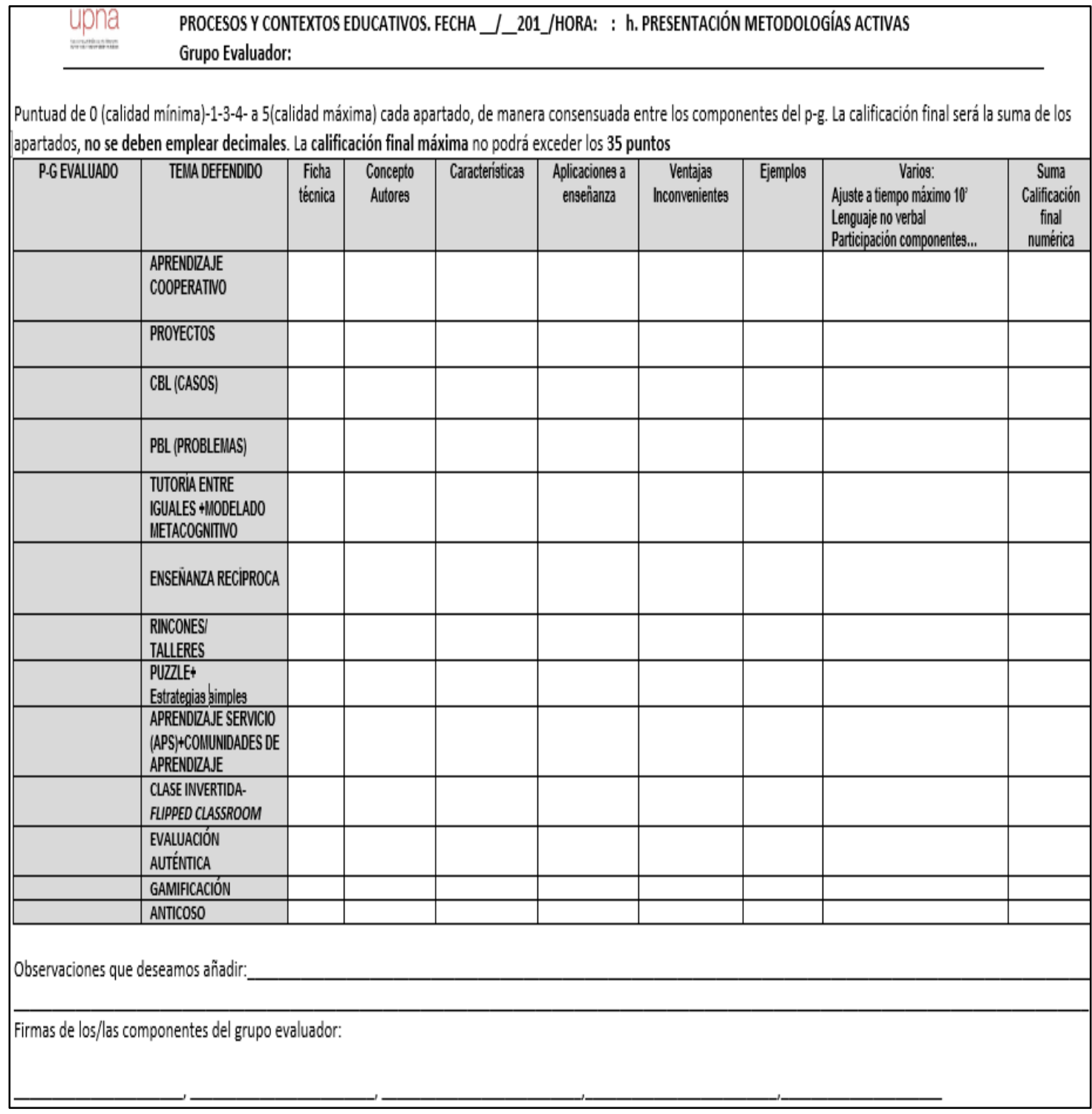

Figura 3. Escala de estimación Metodologías activas

Fuente: Elaboración propia.

Pero también existe la posibilidad de que cada $\mathrm{p}-\mathrm{g}$ realice observaciones, reflexiones, sugerencias, justificaciones que permiten una evaluación formativa. Igualmente, la docente realiza un feedfodward tanto durante la exposición oral como con la corrección 
del documento-síntesis de estudio, que cada p-g debe revisar y reformar antes de ser subido a la plataforma intranet MiAulario, de modo que esté disponible para todo el grupo aula de cara a trabajos posteriores de aplicación de las diversas metodologías y también para constituirse como materia de evaluación sumativa en prueba escrita final. Tras cada sesión, la docente publica además en la intranet una gráfica con las puntuaciones medias para que cada p-g pueda mejorar de cara a próximas exposiciones orales en los diversos aspectos evaluados o pedir tutoría para encontrar estrategias de optimización (ver Figura $4)$.

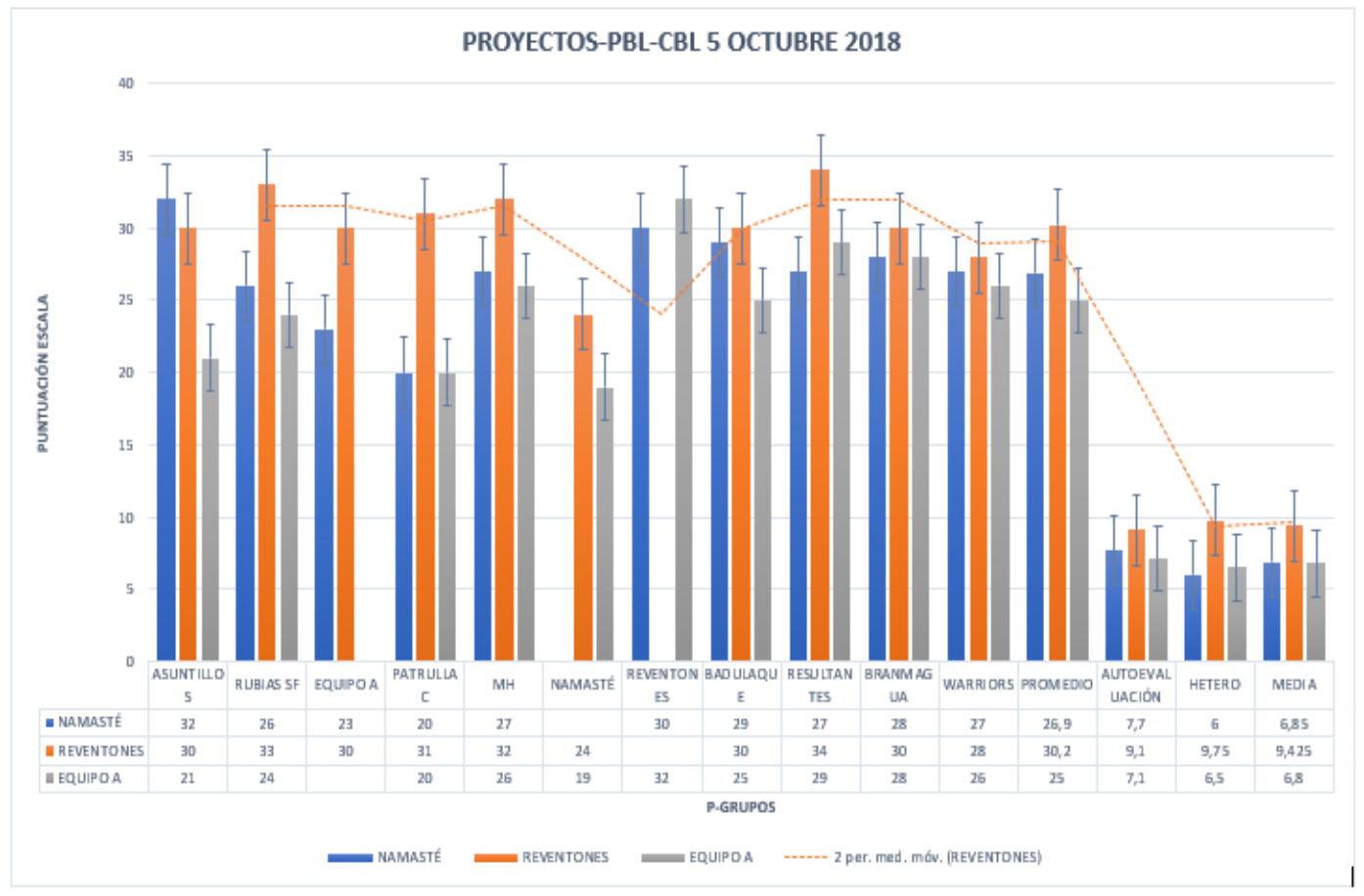

Figura 4. Ejemplo: Gráfica de valores medios Coevaluación y Heteroevaluación Fuente: Elaboración propia.

En tiempo simultáneo al desarrollo de las actividades en aula universitaria, los alumnos y alumnas ya se han incorporado a las aulas de los colegios, por lo que han hecho un primer diagnóstico de necesidades o han recibido solicitudes de maestros y maestras para que diseñen propuestas incluyendo alguna metodología activa. Uno de los objetivos que persigue esta formación dual es atender a las necesidades de los colegios para innovar sus métodos de enseñanza-aprendizaje de modo que puedan responder a la diversidad del alumnado y gestionar grupos con altas ratios por aula.

El Criterio de Complejidad de la acción competente supone saber interpretar las situaciones (analizarlas y sistematizar sus fases) y el criterio de estar compuesta por Factores de índole distinta conlleva articular los principios, las destrezas y las actitudes en un proceso continuo de reflexión del conjunto. Ambos nos hacen abordar el reto de poner al alumnado en situación de realizar un trabajo complejo de programación de unidades didácticas interdisciplinares o globalizadas incorporando metodologías activas, para implementarse en el contexto real de enseñanza-aprendizaje en un aula con escolares de EP. La primera ruptura de esquemas previos se produce en relación al modelo disciplinar de aprendizaje interiorizado por los/las universitarios dada la influencia de toda su historia escolar previa. Supone un gran esfuerzo y renuncia desprenderse de esta 
concepción de la enseñanza, porque, aun siendo denostada, por la memorización excesiva y mecánica que conlleva, aporta seguridad a la hora de afrontar situaciones desconocidas.

A modo de macropráctica estructurada en microtareas, cada p-g cooperativo realiza este trabajo pasando por fases de: 1) acercamiento a elementos prescriptivos del currículo oficial (competencias, objetivos de etapa, áreas de conocimiento con sus bloques de contenido, criterios de evaluación y estándares de aprendizaje evaluables, así como a los elementos transversales), 2) situación en el contexto organizativo, 3) elección de centros de interés temáticos, 4) selección de metodologías y adaptación a las características del aula, 5) elaboración de objetivos didácticos, 6) creación de herramientas de evaluación formativa, entre otros. Un proceso complejo de construcción en bucle que recibe las correcciones de la docente (feedfodward de cada microtarea entregada), que se sintetiza en una videograbación y se defiende ante el grupo-aula siendo evaluada mediante rúbrica (ver cuadro 3).

Cuadro 3. Rúbrica de coevaluación: Defensa oral de U. Didácticas interdisciplinares RÚBRICA EXPOSICIONES ORALES. DEFENSA UNIDADES DIDÁCTICAS. Procesos y contextos educativos

\begin{tabular}{|c|c|c|c|}
\hline EXPOSICIÓN ORAL & CORRECTO (2p) & ACEPTABLE(1p) & INCORRECTO(Op) \\
\hline Claridad & $\begin{array}{l}\text { La exposición se ha hecho } \\
\text { con claridad. Se entiende } \\
\text { perfectamente todo lo } \\
\text { explicado }\end{array}$ & $\begin{array}{l}\text { La exposición ha sido } \\
\text { bastante clara, aunque ha } \\
\text { habido algún aspecto que no } \\
\text { se ha entendido bien }\end{array}$ & $\begin{array}{l}\text { No se ha entendido lo que } \\
\text { se quería explicar o se han } \\
\text { explicado muy mal }\end{array}$ \\
\hline Orden & $\begin{array}{l}\text { Se ha seguido un orden } \\
\text { correcto: presentación, } \\
\text { desarrollo y conclusiones }\end{array}$ & $\begin{array}{l}\text { La explicación no ha sido del } \\
\text { todo ordenada. Podría haber } \\
\text { seguido un orden más } \\
\text { lógico. }\end{array}$ & $\begin{array}{l}\text { La explicación no ha } \\
\text { seguido estructura lógica } \\
\text { ninguna. Se nota que no } \\
\text { estaba preparada. }\end{array}$ \\
\hline $\begin{array}{l}\text { Postura y Contacto } \\
\text { visual }\end{array}$ & $\begin{array}{l}\text { Establece contacto visual } \\
\text { con la audiencia }\end{array}$ & $\begin{array}{l}\text { Alguna vez establece } \\
\text { contacto visual con la } \\
\text { audiencia }\end{array}$ & $\begin{array}{l}\text { No miran a la gente } \\
\text { durante la presentación }\end{array}$ \\
\hline Tiempo & $\begin{array}{l}\text { La presentación se ha } \\
\text { ajustado muy bien al } \\
\text { tiempo preestablecido (10') }\end{array}$ & $\begin{array}{l}\text { Se ha excedido o le falta algo } \\
\text { de tiempo pero no en exceso } \\
\left(12^{\prime}-8^{\prime}\right)\end{array}$ & $\begin{array}{l}\text { Ha terminado muy pronto } \\
\text { o ha utilizado mucho más } \\
\text { tiempo del previsto (no } \\
\text { terminan o se exceden) } \\
\left(15^{\prime}-7^{\prime}\right)\end{array}$ \\
\hline \multicolumn{4}{|l|}{ ELEMENTOS U.D. } \\
\hline $\begin{array}{l}\text { Título, nivel (curso), } \\
\text { momento del curso, } \\
\text { duración ( } n^{\circ} \text { de horas), }\end{array}$ & $\begin{array}{l}\text { El título es motivador y } \\
\text { original y la información } \\
\text { presentada es clara y } \\
\text { completa }\end{array}$ & $\begin{array}{l}\text { El título es atractivo y la } \\
\text { mayoría de datos son } \\
\text { comunicados }\end{array}$ & $\begin{array}{l}\text { El título es poco sugerente } \\
\text { y faltan datos }\end{array}$ \\
\hline Áreas, Competencias & $\begin{array}{l}\text { La selección es } \\
\text { proporcionada }\end{array}$ & $\begin{array}{l}\text { La selección es algo excesiva } \\
\text { o insuficiente }\end{array}$ & $\begin{array}{l}\text { La selección es claramente } \\
\text { excesiva o insuficiente }\end{array}$ \\
\hline $\begin{array}{l}\text { Objetivos } \\
\text { didácticos/criterios de } \\
\text { evaluación-índice de } \\
\text { logro }\end{array}$ & $\begin{array}{l}\text { Los objetivos están } \\
\text { formulados con exactitud y } \\
\text { tienen riqueza de criterios } \\
\text { de evaluación }\end{array}$ & $\begin{array}{l}\text { Los objetivos tienen alguna } \\
\text { inexactitud y los criterios de } \\
\text { evaluación son escasos }\end{array}$ & $\begin{array}{l}\text { Los objetivos están } \\
\text { formulados } \\
\text { inadecuadamente y los } \\
\text { criterios de evaluación son } \\
\text { poco concretos }\end{array}$ \\
\hline $\begin{array}{l}\text { Síntesis de actividades, } \\
\text { aplicación de } \\
\text { metodología cooperativa }\end{array}$ & $\begin{array}{l}\text { Las actividades son } \\
\text { originales, con riqueza y } \\
\text { variedad de recursos, } \\
\text { favorecen frecuentemente } \\
\text { la creatividad y la } \\
\text { participación del alumnado }\end{array}$ & $\begin{array}{l}\text { Las actividades están bien } \\
\text { diseñadas, emplean recursos } \\
\text { habituales, permiten la } \\
\text { creatividad y ocasionalmente } \\
\text { la participación del } \\
\text { alumnado }\end{array}$ & $\begin{array}{l}\text { Las actividades son poco } \\
\text { originales, los recursos } \\
\text { escasos y sin variedad, no } \\
\text { fomentan la creatividad y } \\
\text { no favorecen la } \\
\text { participación del } \\
\text { alumnado }\end{array}$ \\
\hline $\begin{array}{l}\text { Evaluación del } \\
\text { aprendizaje y de la } \\
\text { enseñanza }\end{array}$ & $\begin{array}{l}\text { Los instrumentos, } \\
\text { momentos y agentes de } \\
\text { evaluación, coevaluación y } \\
\text { heteroevaluación quedan } \\
\text { explicitados claramente }\end{array}$ & $\begin{array}{l}\text { Los instrumentos, } \\
\text { momentos y agentes de } \\
\text { evaluación, coevaluación y } \\
\text { heteroevaluación son } \\
\text { referidos con algún grado de } \\
\text { claridad }\end{array}$ & $\begin{array}{l}\text { Los instrumentos, } \\
\text { momentos y agentes de } \\
\text { evaluación, coevaluación y } \\
\text { heteroevaluación se } \\
\text { refieren de manera confusa }\end{array}$ \\
\hline Valoración final & $\begin{array}{l}\text { Las referencias son } \\
\text { reflexivas y constructivas }\end{array}$ & $\begin{array}{l}\text { Las referencias son en parte } \\
\text { reflexivas }\end{array}$ & $\begin{array}{l}\text { Las ideas son negativas y } \\
\text { escasamente reflexivas }\end{array}$ \\
\hline PUNTUACIÓN & & & \\
\hline
\end{tabular}

Fuente: Elaboración propia. 
La implementación de la propuesta didáctica interdisciplinar con metodologías activas es valorada, por una parte por los maestros y maestras del aula escolar quiénes van a llevar a cabo la evaluación de todo el proceso de formación dual vivido desde los colgios. El reflejo del grado de consecución de aprendizaje se lleva a cabo a nivel cualitativo en un documento acreditativo que cada estudiante puede adjuntar a su Curriculum Vitae. Cada alumno y alumna cumplimenta su autoevaluación en una Escala de Valoración de Competencias en tres niveles y que se adjunta en el reverso del Informe (ver cuadro 6 y cuadro 7 en Anexo I).

Además de realizar una prueba objetiva clásica, con formato de test, que contribuye a la valoración de las dimensiones más conceptuales de la asignatura, cada alumno y alumna, de manera individual auto y coevalúa el funcionamiento como grupo cooperativo y lo envía por email (ver cuadro 4).

Cuadro 4. Ficha individual de auto y coevaluación del funcionamiento p-g.

\begin{tabular}{lll}
\hline $\begin{array}{c}\text { COMPONENTES DEL P-G } \\
\text { COOPERATIVO }\end{array}$ & VALORACIÓN CUALITATIVA & \multicolumn{1}{c}{$\begin{array}{c}\text { VALORACIÓN } \\
\text { CUANTITATIVA (DE 0-10) }\end{array}$} \\
\hline YO & -Implicación & p.ej. \\
NOMBRE Y APELLIDOS & $\begin{array}{l}\text {-Aportaciones/iniciativas } \\
\text {-Responsabilidad/compromiso } \\
\text {-Actitudes de compañerismo }\end{array}$ & $\begin{array}{l}\text { Una cifra única global que } \\
\text { tenga en cuenta los } \\
\text { diferentes aspectos }\end{array}$ \\
Nombre p-g: & $\begin{array}{l}\text {-Puntualidad } \\
\text {-Originalidad }\end{array}$ & \\
\hline Alumno/Alumna 1 & -Otros... & \\
NOMBRE Y APELLIDOS & -Implicación & \\
& -Aportaciones/iniciativas & \\
(aumenta una casilla para & -Responsabilidad/compromiso & \\
cada componente del p-g) & -Puntualidad & -Originalidad \\
… & -Otros... & \\
\hline \multirow{2}{*}{ Deseo añadir.... } & & \\
\hline
\end{tabular}

Fuente: Elaboración propia.

En el cuadro contiguo se recogen, de manera sintética, tanto las actividades formativas realizadas en los contextos de aula universitaria y aula escolar, de manera simultánea, como los momentos de participación de los diversos agentes en la auto, co y hetereroevaluación, de modo que van teniendo lugar los procesos de Feedback y de Feedforward en un continuum que permite el desarrollo y evaluación auténtica de las competencias de la asignatura (ver cuadro 5). 
Cuadro 5. Relación entre actividades formativas y medios/técnicas/instrumentos de evaluación

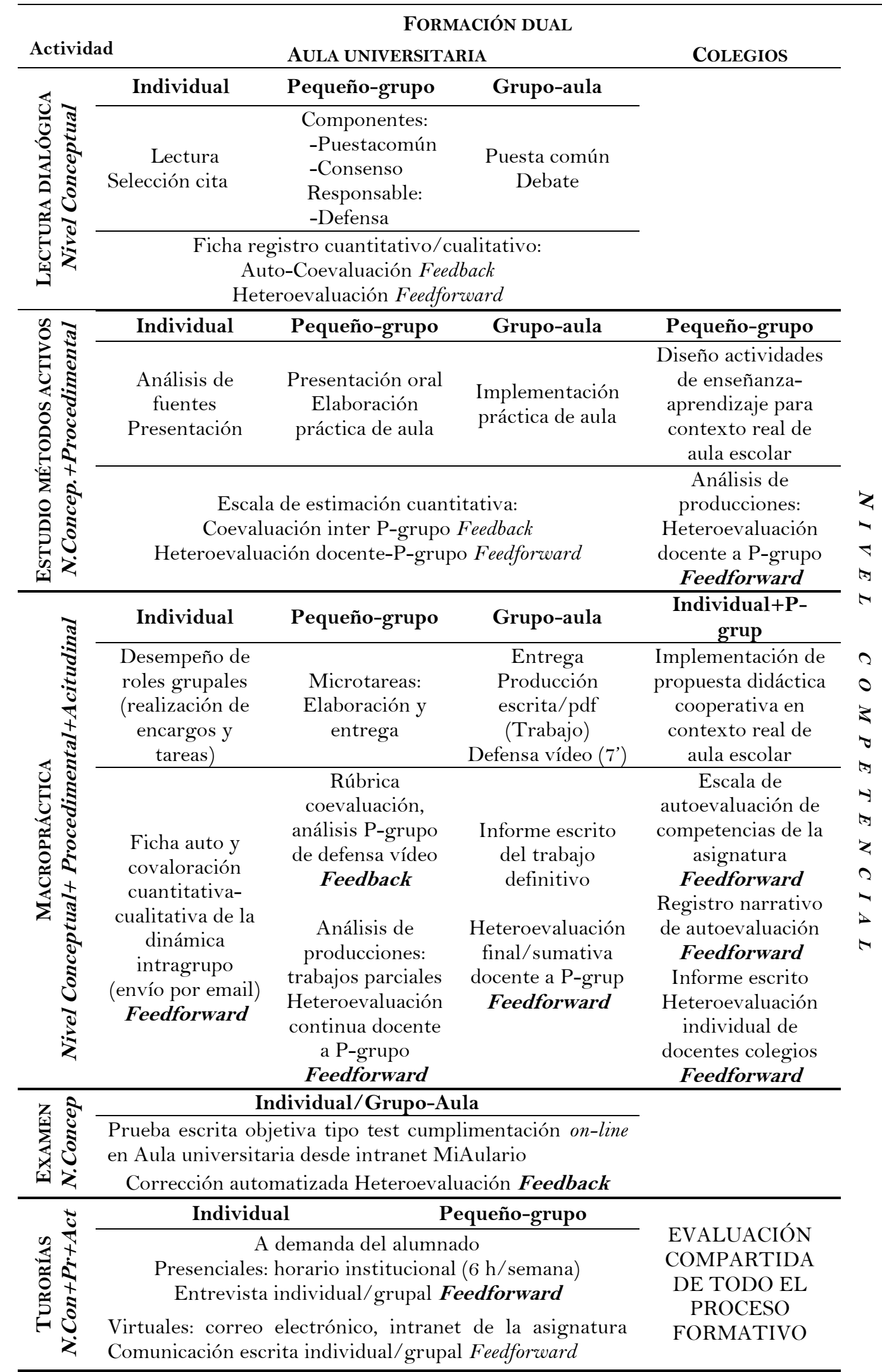

Fuente: Elaboración propia. 


\section{Discusión y conclusiones}

La formación dual en enseñanza superior, en la universidad, es entendida como la formación combinada de dos sistemas: el universitario y el laboral, que en el caso de los Grado de Maestro es el escolar. Sin embargo, no es posible hablar de un único modelo de formación dual, dado que precisa tener en cuenta numerosas y diversas configuraciones en virtud de los enfoques pedagógicos, las líneas didácticas, los contextos organizativos, así como las actividades que se prevé desarrollar en los ámbitos laboral y académico, "considerando las experiencias por separado y en su intersección" (Coiduras, Correa, Boudjaoui y Curto, 2017, p. 85). Por esta razón, la configuración de la propuesta de formación en alternancia que ha emergido en nuestro entorno determinado puede servir como modelo dinámico para otras iniciativas, pero cada una de ellas tendrá que ajustarse a los elementos en intersección mencionados.

La formación dual referida en este trabajo es de tipo exterior asociativa dado que se articula en una sucesión de momentos que se planean como actividades complementarias. Se trata de un diseño universitario, de tipo aplicacionista sobre las metodologías activas que promueven aprendizaje cooperativo, trazando un camino de lógica de la teoría a la práctica. Se reconoce el papel del contexto escolar y de los maestros y maestras, dando cabida a la posibilidad formativa de la interacción entre universidad y colegios de enseñanza primaria. Por esta razón se da una minimización del protagonismo universitario en algunas cuestiones de la praxis, como cuando se implementan en el contexto del aula las actividades interdisciplinares (Bourgeon, 1979; Geay, 1998). Se dan momentos para que el alumnado universitario recupere y utilice lo experimentado en los centros escolares de cara al análisis de lo experimentado a la luz de las concepciones teóricas estudiadas en el aula universitaria. En las aulas de los colegios se da una lógica profesional-docente, entendiendo que la acción formativa es consecuencia de la propia praxis didáctica. La articulación de los escenarios universitario-escolar asegura la formación integral mediante la planificación, la tutorización y el acompañamiento de los y las estudiantes del Grado de Maestro. La práctica es analizada desde los enfoques teóricos y desde éstos se interpreta su efecto.

El escenario de la formación en alternancia se presenta cómo idóneo para la evaluación de competencias ya que implica una acción coordinada partiendo de una secuenciación razonable de tales competencias en niveles de complejidad progresiva y permite al profesorado universitario exigir variadas evidencias de aprendizaje a su alumnado, de modo que éste exhiba sus conocimientos así como las competencias relevantes del perfil profesional en el que se está formando (Cano, 2011). En nuestro caso, la implementación de las actividades de aprendizaje cooperativo en el aula escolar implica las 14 competencias específicas de la asignatura que enmarca la formación dual, destacando la "2. Diseñar, planificar y evaluar los procesos de enseñanza y de aprendizaje atendiendo a criterios interdisciplinares y disciplinares con otros profesionales" (cuadro 1), que con frecuencia es una de las mejor valoradas en el ejercicio reflexivo de autoevaluación de competencias que realiza el estudiante universitario.

La articulación de los procesos de feedback-feedfodward está presente a lo largo de todo el proceso, ya que si bien el objetivo formativo, en su dimensión profunda, precisa de una actitud mantenida de reflexión sobre el aprendizaje y de una reubicación en puntos de continua mejora (Cano, 2015), es cierto que tiene que construirse en un medio (el universitario), en el que las inercias hacia la obtención de juicios externos sobre el ‘bien’ o 
'mal' de la acción es requerida e incluso añorada por los estudiantes, en aras de obtener una pretendida seguridad derivada del planteamiento más academicista, y dominante todavía, en el escenario de la educación superior e incluso en las etapas educativas que le preceden, tal y como apunta Robinson (2015). El camino de transición de una vivencia de la evaluación ceñida al feedback de aprobación y acreditación, requiere del diseño de herramientas de recogida de información (fichas de autorregistro, escalas estimativas, rúbricas de coevaluación) pero no se ciñe a la disponibilidad de tales soportes. El verdadero cambio deviene de la transformación de actitudes que paulatinamente van teniendo los sujetos implicados, alumnado y profesorado, y que se va evidenciando en una progresiva implicación, un empleo más riguroso de la recolección de datos y una satisfacción inherente a la experiencia de mejora, afectando tanto a las dimensiones conceptuales como aquellas más afectivas de una acción competente (Zabala y Arnau, 2008)

A modo de conclusión, tomamos como referencia las palabras de Murillo, MartínezGarrido y Belavi (2017, p. 30) cuando afirman que "Investigamos en Educación para cambiar una realidad que nos rodea y que nos duele, investigamos para que se tomen mejores decisiones en la política educativa, en el centro docente o en el aula (...) para construir una mejor sociedad", este es el planteamiento de nuestra propuesta de indagación en formación dual, seguir integrando en la formación inicial de los futuros y futuras docentes el necesario cuestionamiento sobre los saberes que les demanda la realidad educativa en la que tendrán que incidir como profesionales. La enumeración de competencias Generales, Transversales y Específicas de los planes de estudios de los Grados universitarios tienden a convertirse en 'trofeos' que se alcanzan al final y se rubrican con las defensas de los TFG. Nada más lejos de la realidad, el camino de 'ser competente' empieza en el aquí y ahora, articulando conocimientos, aplicando principios, tomando decisiones que implican riesgos, etc., por todo ello precisa de escenarios auténticos en los que el desempeño se pueda valorar y optimizar mediante la continua y compartida reflexión para la mejora.

En este sentido, las propuestas que nos planteamos, en aras a seguir dando continuidad a este tipo de formación dual, se orientan en primer lugar, a la construcción de una cultura institucional de verdadera colaboración entre la universidad, los colegios y la administración educativa; en segundo lugar a definir y depurar los procedimientos de evaluación en la dimensión competencial de los saberes; en tercer lugar, en crear redes de profesorado de los distintos niveles formativos, que sirva para retroalimentar tanto las buenas prácticas como la reflexión profunda sobre los perfiles profesionales necesarios, no sólo para dar respuesta a las demandas sociales sino para anticiparlas y ser motores de innovación.

\section{Referencias}

Apalategi, J. y Gabari, M. I. (2018). Relación de dualidad entre la realidad profesional docente y el aula universitaria: implicaciones en la autonomía universitaria. En I. del Arco y P. Silva (Eds.), Tendencias nacionales e internacionales en la organización educativa: entre la estabilidad y el cambio (pp. 35-340). Madrid: Wolters Kluwer

Bauman, Z. (2015). Modernidad líquida. Ciudad de México: Fondo de Cultura Económica.

Beraza, J. M. y Azkue, I. (2018). Diseño de un itinerario de formación dual: universidad-empresa en GADE. Journal of Management and Business Education, 1(1), 53-68. 
Bourgeon, G. (1979). Socio-pédagogie de l'alternance. París: UNMFREO.

Cano, E. (2015). Evaluación por competencias en educación superior. Madrid: La Muralla.

Cano, E. (2011). Buenas prácticas en la evaluación de competencias. Cinco casos de educación superior. Barcelona: Laertes.

Coiduras, J. L., Correa, E., Boudjaoui, M. y Curto, A. (2017). Formación dual en el Grado de Educación: Claves organizativas y pedagógicas. Revista Qurriculum, 30, 81-102.

Colmenares, A. M. y Piñero, M. L. (2008). La investigación acción. Una herramienta metodológica heurística para la comprensión y transformación de realidades y prácticas socio-educativas. Año, 14(27), 96-114.

Comisión-Europea. (2017). Documento de reflexión sobre la dimensión social de europa. Bruselas: Oficina de Publicaciones.

Consejo Comisión Europea. (2018). Recomendación del Consejo, de 22 de mayo de 2018, relativa a las competencias clave para el aprendizaje permanente. Recuperado de: https://eurlex.europa.eu/legal-content/ES/TXT/PDF/?uri=CELEX:32018H0604(01)\&from=ES

Espinoza Freire, E., Tinoco Izquierdo, W. E. y Sánchez Barreto, X. del R. (2017). Características del docente del siglo XXI. Olimpia: Publicación Científica de La Facultad de Cultura Física de La Universidad de Granma, 14(43), 39-53.

Fernández, J. T. y Bueno, C. R. (2016). Evaluación de competencias profesionales en educación superior: Retos e implicaciones. Educacion XX1, 19(1), 17-38. https://doi.org/10.5944/educXX1.12175

Geay, A. (1998). L'école de l'alternance. París: L'Harmattan.

Ion, G., Silva, P. y Cano, E. (2013). El feedback y el feedforward en la evaluación de las competencias de estudiantes universitarios. Profesorado. Revista de Currículum y Formación Del Profesorado, 17(2), 283-301.

Le Boterf, G. (2018). Développer et mettre en auvre la compétence: Comment investir dans le professionnalisme et les compétences. París: Eyrolles.

Lemenu, D., y Heinen, E. (2015). Comment passer des compétences à l'évaluation des acquis des étudiants? Louvain-la-Neuve: de Boeck.

Lemonnier, M. (2019). Le testamente de Zygmunt Bauman. L'OBS, 2837, 72-73.

Loisy, C. (2016). Desarrollo profesional y construcción de la función docente. Lyon: Universidad de Lyon.

Murillo, F. J., Martínez-Garrido, C. y Belavi, G. (2017). Sugerencias para escribir un buen artículo científico en educación. REICE. Revista Iberoamericana sobre Calidad, Eficacia y Cambio en Educación, 15 (3), 5-34. http://doi.org/10.15366/reice2017.15.3.001

Pascual-Arias, C., López-Pastor, V. M. y Hamodi-Galán, C. (2019). Proyecto de innovación docente : La evaluación formativa y compartida en educación. Resultados de transferencia de conocimiento entre universidad y escuela. Revista Iberoamericana de Evaluación Educativa, $12(1), 29-45$.

Robinson, K. (2015). Escuelas creativas. La revolución que está transformando la educación. Barcelona: Grijalbo.

Tejada F, J. (2012). La alternancia de contextos para la adquisición de competencias profesionales en escenarios complementarios de educación superior: Marco y estrategia. Educacion XX1, 15(2), 17-40. https://doi.org/10.5944/educxx 1.15.2.125

Zabala, A. y Arnau, L. (2008). La enseñanza de las competencias. Innovación Educativa, 161, 40-46. 
Zabala, A. y Arnau, L. (2014). Métodos para la enseñanza de las competencias. Barcelona: Graò.

\section{Anexo I}

Cuadro 6. Informe de tutora escolar

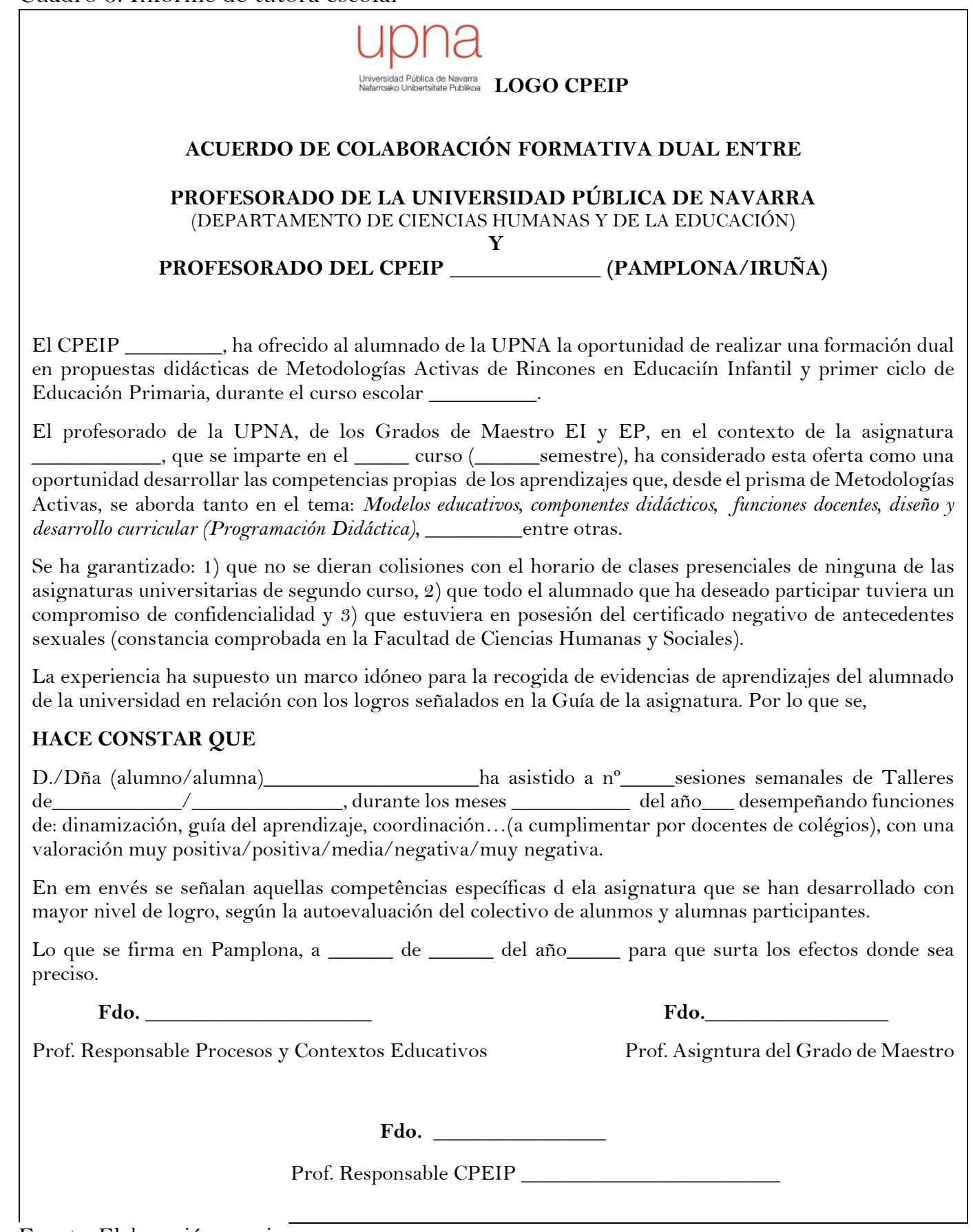

Fuente: Elaboración propia. 
Cuadro 7. Escala autoevaluación competencias

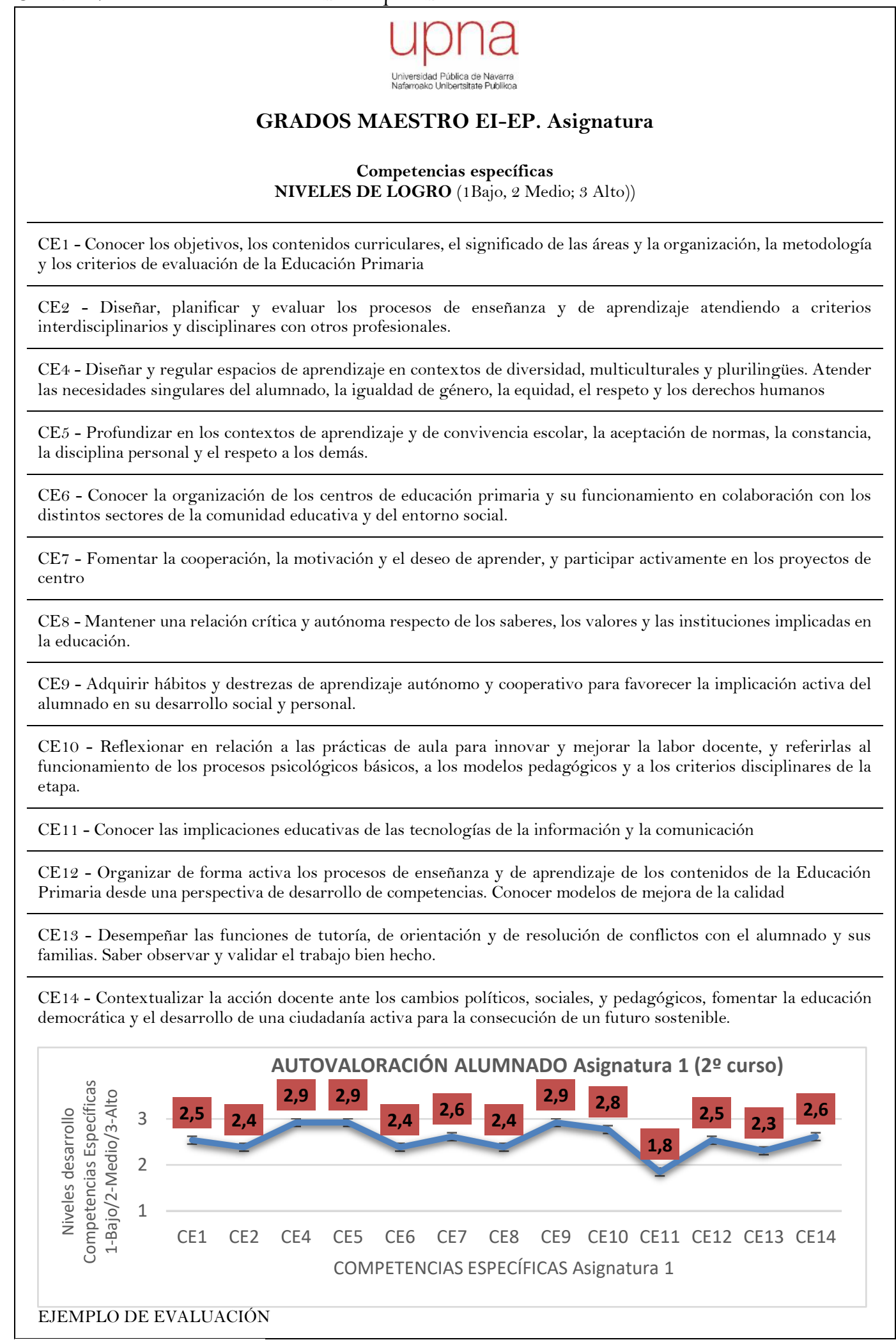

Fuente: Elaboración propia. 


\section{Breve CV de las autoras}

\section{Inés Gabari Gambarte}

Doctora en Ciencias de la Educación. Profesora Titular de Universidad del Área de Didáctica y Organización Escolar, en estudios de Grado, Máster y Doctorado Responsable del Grupo de investigación 'Educación y Salud' (UPNA). Autora/ coautora de comunicaciones, symposios, póster y ponencias invitadas en encuentros científicos de alcance local, regional, nacional e internacional, en Europa (Francia, Suecia, Reino Unido o Portugal), en Latinoamérica (México, Colombia o Argentina) y Estados Unidos (Orlando, Florida). Autora/coautora de publicaciones de libros y artículos en revistas nacionales e internacionales. Participación en Proyectos de investigación subvencionados (como IP y miembro de equipo). Directora/codirectora de trabajos científicos: Tesis doctorales y Trabajos Fin de Máster. Miembro de Tribunales, comités científicos y revisora en revistas científicas. ORCID ID: 000-0002-1226-8819. Email: igabari@unavarra.es

\section{Jaione Apalategi Begiristain}

Licenciada en Pedagogía por la Universidad de Deusto en 1982. Doctora en Ciencias de la Educación en 1994 por la UPV/EHU. Profesora Titular. Docente en los Grados de Maestro en Educación Infantil, Educación Primaria y en el Máster Universitario en Profesorado de Educación Secundaria e investigadora en el ámbito del área de didáctica y organización educativa y dilatada experiencia en gestión de la Facultad de Ciencias Humanas y Sociales como Vicedecana y Decana de la misma. Miembro del Patronato de la Fundación Museo Jorge Oteiza Fundazio Museoa desde su creación en 1992 con sede en Alzuza, Navarra (www.museooteiza.org). ORCID ID: 0000-0002-3599-5859. Email: jaione.apalategi@unavarra.es 